\title{
Cardiac allograft vasculopathy in Dutch heart transplant recipients
}

\author{
G. Galli ${ }^{1,2}$ • K. Caliskan ${ }^{1}$ - A. H. M. M. Balk ${ }^{1}$ R. van Domburg ${ }^{1}$ - O. Birim ${ }^{3}$ J. Salerno-Uriarte ${ }^{2}$. \\ O. C. Manintveld ${ }^{1}$ A. A. Constantinescu ${ }^{1}$
}

Published online: 1 September 2016

(c) The Author(s) 2016. This article is available at SpringerLink with Open Access.

\begin{abstract}
Background Cardiac allograft vasculopathy (CAV) is a multifactorial disease and a major cause of graft failure after heart transplantation. However, the impact of CAV may vary according to the definition and the regional differences in transplantation settings.

Objectives We sought to assess CAV prevalence, predictors and prognosis in Dutch heart transplant recipients based on coronary angiography, following the 2010 standard nomenclature of the International Society for Heart and Lung Transplantation.

Methods Patients $\geq 18$ years who underwent heart transplantation at our centre with at least one coronary angiography during follow-up were included in the analysis. Clinical variables were collected prospectively.

Results Among 495 analysed recipients, there were 238 (48\%) with CAV. The prevalence of CAV was 18, 47 and $70 \%$ at 4,12 and 20 years, respectively. In the multivariable proportional hazards regression analysis, only male donor gender and increasing donor age were significantly associated with the risk of CAV. The long-term prognosis of the patients with CAV at fourth-year angiography was significantly worse as compared with that of CAV-free patients, independently of the severity of CAV $(p<0.001)$.
\end{abstract}

A. A. Constantinescu

a.constantinescu@erasmusmc.nl

1 Department of Cardiology, Erasmus MC, Rotterdam, The Netherlands

2 Department of Cardiology, University of Insubria, Ospedale di Circolo and Fond. Macchi, Varese, Italy

3 Department of Cardiothoracic Surgery, Erasmus MC, Rotterdam, The Netherlands
Conclusion The prevalence of CAV increased gradually over time, with a similar trend as in other registries. Posttransplant survival is decreased in patients with any degree of early CAV, indicating that management strategies should start with donor selection and preventive measures immediately after transplantation.

Keywords Cardiac allograft vasculopathy (CAV) - Donor age $\cdot$ Heart transplantation prognosis

\section{Introduction}

Cardiac allograft vasculopathy (CAV) is one of the major causes of late graft failure and death in heart transplant patients [1]. The reported CAV prevalence varies according to the definition, population, transplantation period and follow-up protocol and ranges from 39 to $65 \%$ at 10 years in single-centre studies, while in the large register of the International Society for Heart and Lung Transplantation (ISHLT) it is $50 \%$ at 10 years [1-3]. CAV is characterised by concentric thickening of the wall of large and small coronary vessels and has various histological patterns, including inflammatory lesions, lesions rich in smooth muscle cells and fibrotic lesions, which have been related to the time passing after transplantation $[4,5]$. The pathogenesis of CAV has been related to immunological and non-immunological factors in both the donor and the recipient, but the exact triggers and the pathophysiological pathways are still unknown $[6,7]$. The data are heterogeneous due to different transplantation decades, different populations and treatment protocols and their generalisability is further hampered by various diagnostic criteria of CAV [6, 8]. Standardisation of the CAV diagnosis and gradation was recommended in 
2010 by the ISHLT based on conventional coronary angiography [9].

In the Netherlands, the shortage of the donors has led to an increase in the mean donor age from 29 to 43 years, while the most frequent cause of death shifted from trauma to stroke. Despite the use of older donors, we found an improved survival after heart transplantation in the last decade at our centre [10]. However, subclinical atherosclerosis may be more frequent in donor hearts from older patients with neurovascular comorbidity and, therefore, the first aim of the current study was to investigate CAV prevalence and predictors in the patients undergoing heart transplantation in the Netherlands, using our large single-centre cohort. Secondly, we aimed to assess the long-term prognosis taking into account the diagnosis and severity of CAV.

\section{Patients and methods}

\section{Study population}

Since the first orthotopic heart transplantation at our centre in June 1984, data of all heart transplant recipients were collected prospectively until December 2012. Patients consented to the use of anonymised data for research purposes. The institutional review board of the Erasmus MC approved the present study.

Only patients $\geq 18$ years who underwent at least one conventional coronary angiography at follow-up were included in the analysis. We recorded recipient-related and donorrelated variables based on the clinical relevance and previously published studies on CAV predictors. Recipient pretransplant clinical variables were age, gender, aetiology of heart failure, creatinine and diabetes. Donor-related data were age, gender and cause of death. Donor-recipient mismatch variables and available immunological information were collected. Data at one year after transplantation included the number of acute rejection episodes, development of cytomegalovirus-related disease, serum creatinine, total cholesterol, triglycerides, diagnosis of hypertension and diabetes. Rejection surveillance was based on endomyocardial biopsies, which were graded according to the Billingham's criteria until $2004[11,12]$ and subsequently according to the ISHLT revised guidelines [13]. Acute rejections were defined as the treated rejections within the first year after transplantation in each patient. Immunosuppressive medication and the use of statins were recorded at the time of CAV diagnosis or at the time of the most recent angiography for the patients without CAV. Mortality and retransplantation were recorded as outcome events.

\section{Diagnosis of CAV}

Coronary angiography (CAG) was performed per protocol every year until 1990. After 1990, CAG was performed at one year and repeated every year in case of evidence of CAV, otherwise postponed to the fourth year after transplantation [14]. After the fourth year post-transplantation, patients underwent non-invasive myocardial perfusion scintigraphy annually for assessment of ischaemia. CAG was performed in these patients when perfusion scintigraphy was positive, or when ischaemia was suspected based on cardiac markers or clinical, electrocardiographic or echocardiographic criteria. Two cardiologists reviewed all the available coronary angiographies, or, when the CAG images were absent in our archive, the diagnosis of CAV was made from the written report and re-graded according to the 2010 ISHLT criteria [9]. In summary, CAV grade 1 applies to stenosis $<70 \%$ of primary or secondary coronary branches, CAV grade 2 applies to stenosis $\geq 70 \%$ of one primary branch or two secondary branches, CAV grade 3 applies to stenosis $\geq 70 \%$ of two primary branches or three secondary branches, or if an impaired left ventricular function is associated with any degree of CAV. For each patient we recorded the time from heart transplant until the first CAG showing any degree of CAV.

\section{Statistical analysis}

For the analysis of CAV prevalence, the proportion of patients with CAV among survivors was calculated at predefined points, i.e. at $1,4,8,12,16$ and 20 years posttransplantation. Donor and recipient variables were compared between CAV-free and CAV patients. Categorical data are presented as numbers with percentages. Continuous data are presented as mean \pm standard deviation. The data were compared using the chi-square test for categorical variables, and independent sample t-test for continuous variables. Univariate analysis of donor and recipient characteristics was performed using the Cox proportional hazards model. Significant results from the univariate analysis $(p \leq 0.05)$, but also non-significant clinically relevant variables were included in the multivariable proportional hazards regression analysis using the backward procedure. For survival analysis, the outcome was the combination of allcause mortality and retransplantation according to the presence or absence of CAV using the Kaplan-Meier method and the log-rank test for comparison. Data were analysed using IBM SPSS Statistics version 21. 
Fig. 1 Flowchart study population

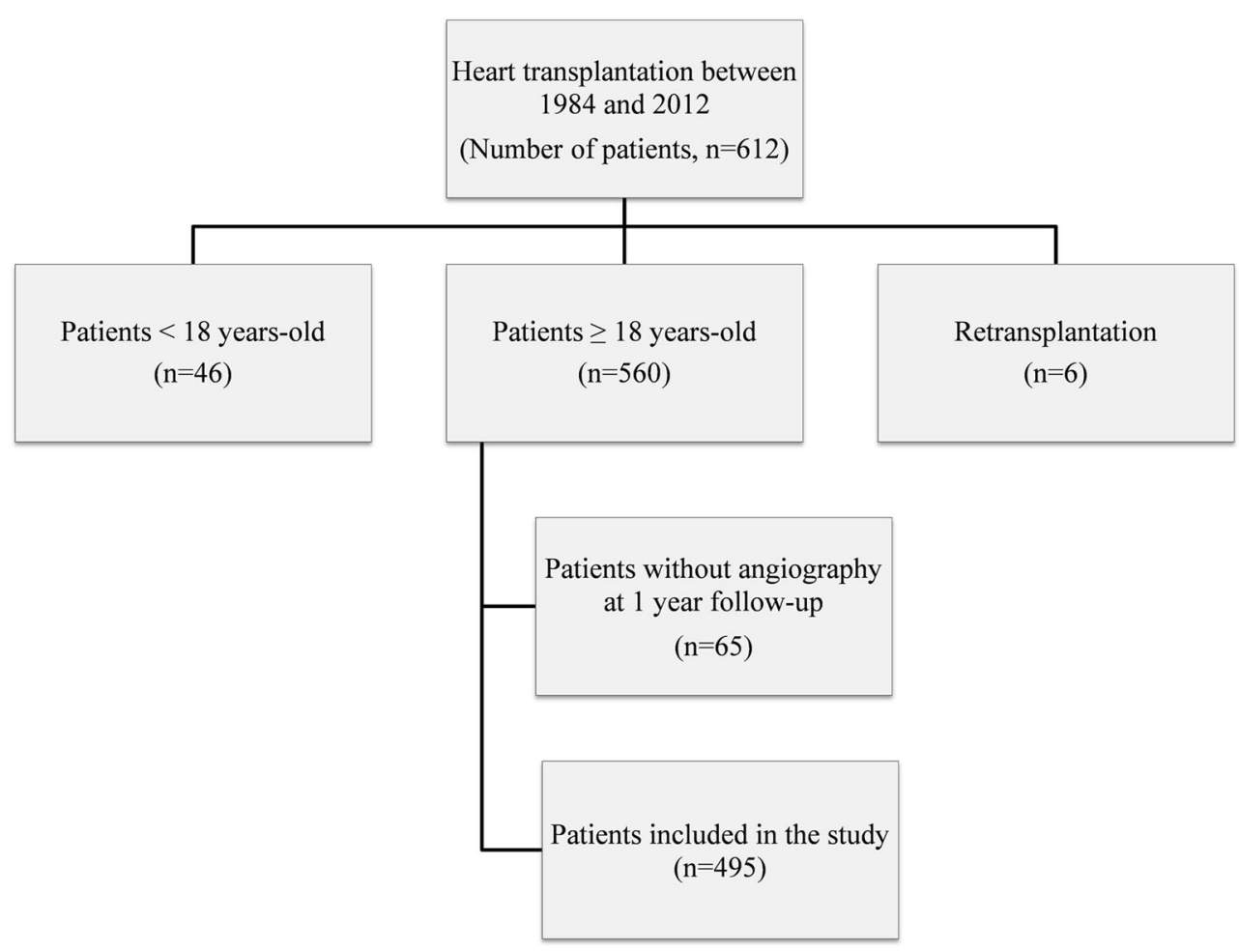

Table 1 Detection of CAV after heart transplantation

\begin{tabular}{ll}
\hline & $N(\%)$ \\
\hline Mean follow-up & $10.6 \pm 5.7$ years \\
$N$. patients with CAV diagnosis & $238(48.1)$ \\
Time to CAV after heart transplantation & $6.1 \pm 4.2$ years \\
Median & 4.9 years \\
Range & 17 days-18 years \\
CAV grade at diagnosis & - \\
1 & $143(60.1)$ \\
2 & $65(27.3)$ \\
3 & $30(12.6)$ \\
\hline
\end{tabular}

\section{Results}

From the cohort of 612 heart transplant recipients, 495 were included in the analysis (Fig. 1). The mean age at transplantation was $48.6 \pm 10.3$ years, and $77.4 \%$ of recipients were males. Ischaemic heart disease was the cause of heart failure before transplantation in $49.9 \%$ of the patients. Diabetes was present in $6.1 \%$ of the patients before transplantation. Mean donor age was $33.3 \pm 12.9$ years and $52.7 \%$ of the donors were males. The cause of death was trauma in $48.8 \%$ of the donors. During the first year post-transplantation, hypertension, diabetes and cytomegalovirus disease were present in 75, 32 and $20 \%$ of the patients, respectively. At one year after transplantation only a minority of the recipients $(22.6 \%)$ were free of any rejection episodes, while $21.6 \%$ had more than two rejection episodes.

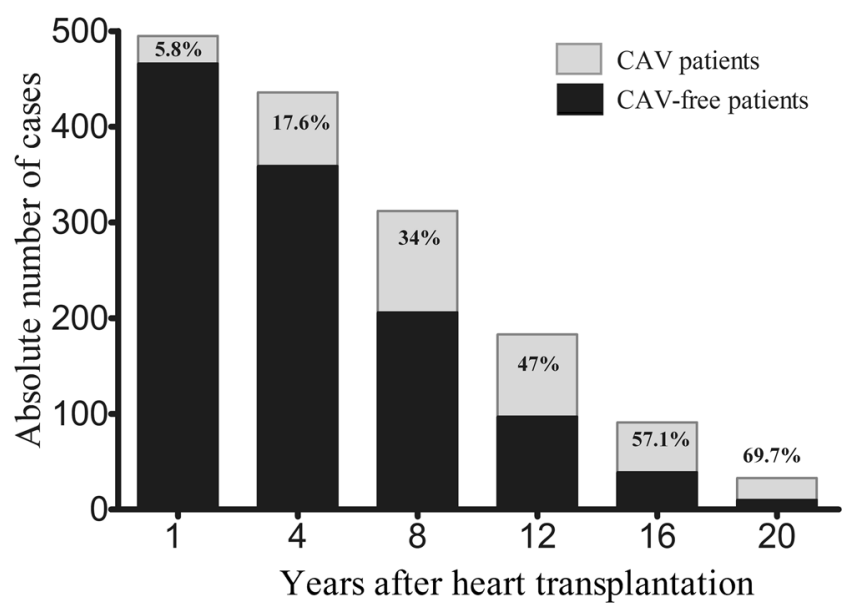

Fig. 2 CAV prevalence in survivors after heart transplantation

A total of 238 (48.1\%) transplant recipients received the diagnosis of CAV. Mean time to CAV detection was $6.1 \pm$ 4.2 years after transplant, with the median at the fourth year after transplantation, when CAG was routinely performed according to the follow-up protocol. The majority of the affected patients $(60.1 \%$ ) had a mild CAV (grade 1), while $12.6 \%$ of the patients had severe CAV (grade 3 ) at diagnosis (Table 1). The prevalence of CAV increased gradually from $17.6 \%$ at 4 years to $47 \%$ at 12 years and $69.7 \%$ at 20 years, while the number of surviving transplant recipients decreased during the follow-up period (Fig. 2).

The recipient characteristics before and after transplantation, donor characteristics, immunosuppression and statin 


\section{Advertisement placed here.}

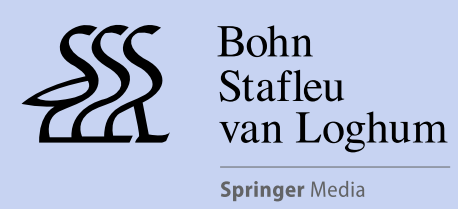

Houten 2016 


\section{Advertisement placed here.}

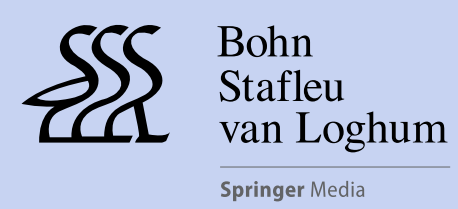

Houten 2016 
Fig. 3 Forest plot of risk factors included in the multivariate Cox proportional hazard modelling

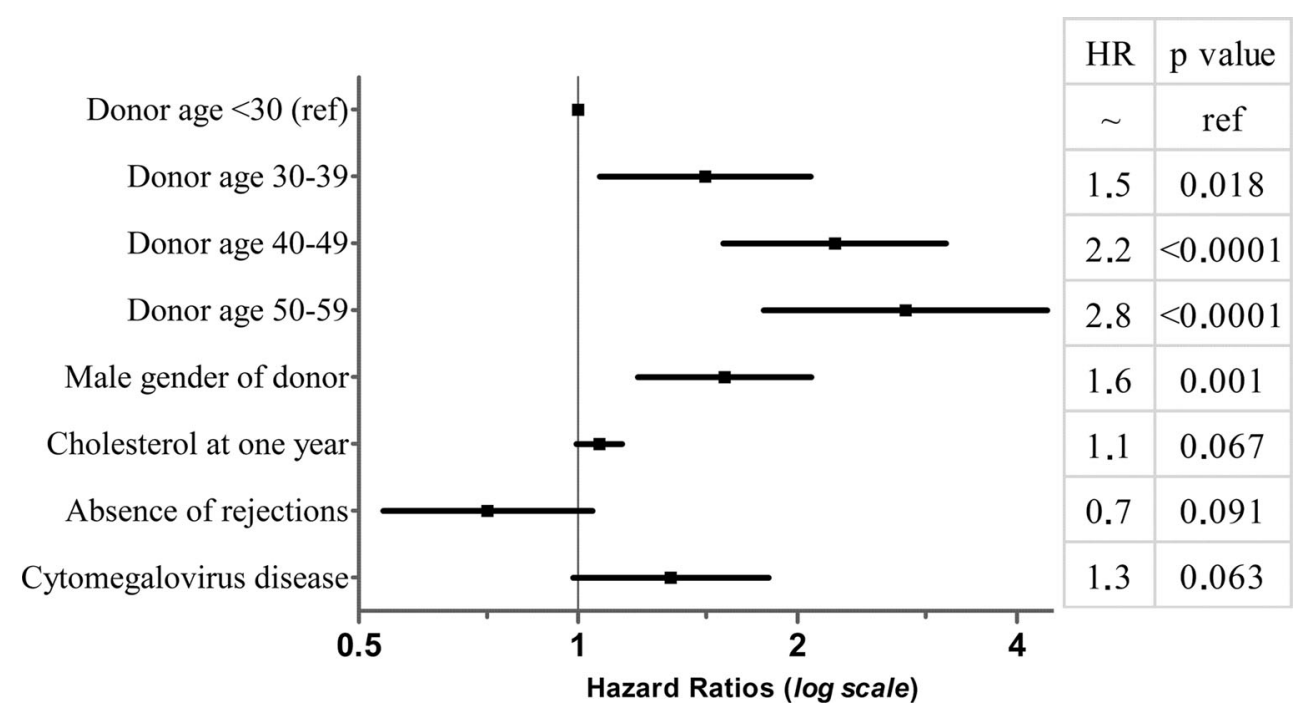

a

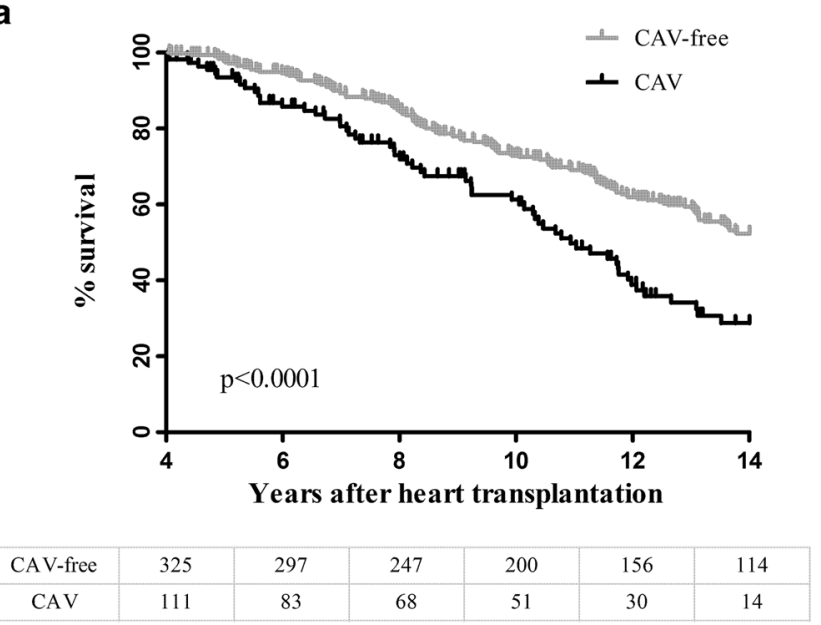

b

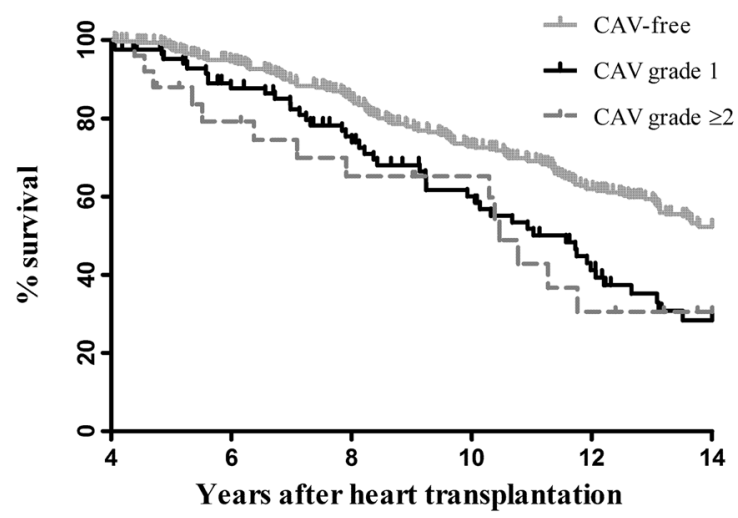

\begin{tabular}{|c|c|c|c|c|c|c|}
\hline CAV-free & 325 & 297 & 247 & 200 & 156 & 114 \\
\hline CAV 1 & 86 & 70 & 53 & 38 & 24 & 12 \\
\hline CAV2 and 3 & 25 & 13 & 15 & 13 & 6 & 2 \\
\hline
\end{tabular}

Fig. 4 a Long-term survival in CAV versus CAV-free patients. Kaplan-Meier analysis used to compare survival of patients alive at four years, grouped into patients with CAV diagnosis within the fourth year and CAV-free patients. Curves were compared with log-rank test. b Long-term survival according to CAV severity at four years. Log-rank overall: $\mathrm{p}<0.0001$, while log-rank analysis between CAV1 versus CAV $\geq 2$ was not significant $(\mathrm{p}=0.0656)$

therapy were compared between the cohorts of patients with CAV and without CAV (Table 2). a significant difference in the recipient age at transplantation and the donor age was seen between the two cohorts. There was a significantly higher proportion of male donors and higher cholesterol levels at one year in the CAV cohort as compared with the CAV-free cohort. No significant difference was found in the immunosuppression induction therapy or maintenance immunosuppression or statins therapy at the moment of CAV diagnosis, renal function or the presence of diabetes between the two cohorts. There was a trend for a larger proportion of patients without any rejection in the CAVfree cohort as compared with the CAV cohort $(p=0.06)$.

In the multivariable proportional hazard regression analysis only male donor gender and donor age were significantly associated with $\mathrm{CAV}$, with a gradual increased hazard risk of 1.5, 2.2 and 2.8 in the three consecutive groups of donor age, while post-transplantation clinical factors were not significant (Fig. 3). The treatment with mycophenolate mofetil was associated with a significantly increased risk (HR $1.4, p=0.008$ ) only in the univariate analysis, while cytomegalovirus disease was associated with an increased hazard risk for CAV (HR 1.3, $p=0.059$ ), although not significant, in the univariate analysis (Table 3 ).

The long-term outcome consisting of survival or retransplantation was compared between the patients with CAV of any degree at fourth-year angiography and the patients with no CAV at the same evaluation (Fig. 4a). The patients with CAV diagnosis had a significantly impaired prognosis as compared with CAV-free patients at the fourth year after transplantation $(p<0.001)$. Also, significantly decreased long-term survival was found for the patients with 
Table 2 Donor and recipient variables in CAV-free and CAV-affected patients

\begin{tabular}{|c|c|c|c|}
\hline & $\begin{array}{l}\text { CAV-free population, } \\
\text { Group I }(n=257), N(\%)\end{array}$ & $\begin{array}{l}\text { CAV population, } \\
\text { Group II }(n=238), N(\%)\end{array}$ & $p$ value $^{\mathrm{a}}$ \\
\hline \multicolumn{4}{|l|}{ Recipient } \\
\hline $\begin{array}{l}\text { Recipient age, years } \\
18-39 \\
40-49 \\
50-59 \\
\geq 60\end{array}$ & $\begin{array}{l}49.7 \pm 10.2 \\
40(15.6) \\
74(28.8) \\
105(40.9) \\
38(14.8)\end{array}$ & $\begin{array}{l}47.4 \pm 10.3 \\
46(19.3) \\
74(31.1) \\
97(40.8) \\
21(8.8)\end{array}$ & $\begin{array}{l}0.01 \\
0.18\end{array}$ \\
\hline Recipient gender: Male/Female & $192 / 65(74.7 / 25.3)$ & $191 / 47(80 / 19.7)$ & 0.11 \\
\hline Previous ischaemic heart disease & $120(46.7)$ & $127(53.4)$ & 0.14 \\
\hline Creatinine pre-heart transplantation $(\mu \mathrm{mol} / \mathrm{l})$ & $121.2 \pm 42.9$ & $121.7 \pm 51.6$ & 0.92 \\
\hline Diabetes pre-heart transplantation & $15(5.8)$ & $15(6.4)$ & 0.81 \\
\hline \multicolumn{4}{|l|}{ Donor } \\
\hline Donor age, years & $32.1 \pm 13.4$ & $34.7 \pm 12.3$ & 0.03 \\
\hline $\begin{array}{l}12-29 \\
30-39 \\
40-49 \\
\geq 50\end{array}$ & $\begin{array}{l}122(48.2) \\
54(21.3) \\
43(17) \\
34(13.4)\end{array}$ & $\begin{array}{l}89(37.9) \\
58(24.7) \\
59(25.1) \\
29(12.3)\end{array}$ & 0.06 \\
\hline Donor death for head trauma & $110(47.4)$ & $105(50.2)$ & 0.53 \\
\hline Donor gender: male/female & $121 / 134(47.5 / 52.5)$ & $140 / 96(59.3 / 40.7)$ & 0.01 \\
\hline \multicolumn{4}{|l|}{ Mismatches donor/recipient } \\
\hline Gender mismatch: donor/recipient & $99(38.8)$ & $83(35.2)$ & 0.4 \\
\hline Cytomegalovirus serology mismatch & $59(23)$ & $55(23.1)$ & 0.97 \\
\hline Toxoplasmosis serology mismatch & $41(16)$ & $41(17.2)$ & 0.7 \\
\hline HLA antibodies mismatch & $69(28.2)$ & $73(31.9)$ & 0.38 \\
\hline \multicolumn{4}{|l|}{ Surgery and medical therapy } \\
\hline Ischaemia time & $170.5 \pm 42.1$ & $176.6 \pm 45.7$ & 0.12 \\
\hline Cyclosporine & $145(56.4)$ & $147(61.8)$ & 0.23 \\
\hline Tacrolimus & $112(43.6)$ & $89(37.4)$ & 0.16 \\
\hline Mycophenolate mofetil (plus tacrolimus) & $65(25.3)$ & $65(27.3)$ & 0.61 \\
\hline Everolimus (plus tacrolimus) & $7(2.7)$ & $10(4.2)$ & 0.37 \\
\hline Statin & $114(44.5)$ & $112(47.1)$ & 0.55 \\
\hline Immunosuppressive induction therapy & $208(80.9)$ & $181(76.1)$ & 0.19 \\
\hline \multicolumn{4}{|l|}{ Parameters at one year after heart transplantation } \\
\hline Cholesterol (mmol/l) & $6.4 \pm 1.7$ & $6.7 \pm 2$ & 0.03 \\
\hline Triglycerides (mmol/l) & $2.3 \pm 1.1$ & $2.3 \pm 1$ & 0.89 \\
\hline Hypertension & $190(73.9)$ & $181(76.1)$ & 0.59 \\
\hline Diabetes & $84(32.7)$ & $76(32.1)$ & 0.88 \\
\hline Creatinine $(\mu \mathrm{mol} / \mathrm{l})$ & $144.5 \pm 79.6$ & $135.4 \pm 59.1$ & 0.14 \\
\hline Cytomegalovirus disease (within first year) & $45(17.5)$ & $55(23.1)$ & 0.12 \\
\hline Absence of rejection episodes & $67(26.1)$ & $45(18.9)$ & 0.06 \\
\hline Total number of rejection episodes & $1.7 \pm 1.6$ & $1.9 \pm 1.6$ & 0.13 \\
\hline
\end{tabular}

All results are expressed as absolute numbers and percentages or mean \pm standard deviation. $P$-values were obtained with Pearson's chi-square test or t-test for equality of means. HLA human leucocyte antigen

mild CAV (grade 1) when compared with CAV-free patients and there was no difference in survival of the CAV grade 1 group when compared with more advanced grades of CAV (Fig. 4b).

\section{Discussion}

Our study has several findings: 1) the prevalence of CAV increased gradually during follow-up to $70 \%$ of the survivors at 20 years; 2 ) donor-related factors were associated with an increased risk of CAV and 3) all CAV severity grades, including mild coronary lesions, at the fourth year 
Table 3 Univariate Cox regression analysis of determinants of CAV

\begin{tabular}{|c|c|c|c|}
\hline & HR & $95 \% \mathrm{CI}$ & $p$ value \\
\hline \multicolumn{4}{|l|}{ Donor age (ref. age $<40$ ) } \\
\hline $40-49$ & 1.4 & $1.04 \pm 2.01$ & 0.03 \\
\hline $50-59$ & 2 & $1.40 \pm 2.72$ & $<0.0001$ \\
\hline$>60$ & 2.2 & $1.45 \pm 3.39$ & $<0.0001$ \\
\hline \multicolumn{4}{|l|}{ Recipient age (ref. age 18-30) } \\
\hline $30-39$ & 1.1 & $0.73 \pm 1.52$ & 0.79 \\
\hline $40-49$ & 1.1 & $0.76 \pm 1.53$ & 0.68 \\
\hline$\geq 50$ & 0.8 & $0.48 \pm 1.35$ & 0.42 \\
\hline Male gender of donor & 1.3 & $0.97 \pm 1.63$ & 0.09 \\
\hline Male gender of recipient & 1.2 & $0.89 \pm 1.68$ & 0.23 \\
\hline Donor death for trauma & 1.2 & $0.92 \pm 1.58$ & 0.18 \\
\hline Previous ischaemic heart disease & 1.3 & $0.98 \pm 1.64$ & 0.07 \\
\hline Creatinine pre-heart transplantation & 1.0 & $1.00 \pm 1.00$ & 0.82 \\
\hline Diabetes pre-heart transplantation & 1.3 & $0.75 \pm 2.13$ & 0.39 \\
\hline Ischaemia time & 1 & $1.00 \pm 1.00$ & 0.77 \\
\hline Toxoplasmosis serology mismatch & 1.1 & $0.82 \pm 1.61$ & 0.42 \\
\hline Cytomegalovirus serology mismatch & 1.1 & $0.78 \pm 1.42$ & 0.74 \\
\hline Cytomegalovirus disease & 1.3 & $0.99 \pm 1.81$ & 0.059 \\
\hline Absence of any episodes of rejection & 0.8 & $0.57 \pm 1.09$ & 0.16 \\
\hline HLA antibodies mismatch & 0.9 & $0.68 \pm 1.19$ & 0.47 \\
\hline Induction treatment & 1.1 & $0.83 \pm 1.51$ & 0.47 \\
\hline Cyclosporine & 0.9 & $0.69 \pm 1.16$ & 0.4 \\
\hline Tacrolimus & 1.1 & $0.83 \pm 1.41$ & 0.55 \\
\hline Mycophenolate mofetil & 1.4 & $1.11 \pm 1.97$ & 0.008 \\
\hline Everolimus & 1.5 & $0.82 \pm 2.20$ & 0.18 \\
\hline Statin & 1.1 & $0.82 \pm 1.37$ & 0.64 \\
\hline Triglycerides (at one year) & 1 & $0.86 \pm 1.09$ & 0.6 \\
\hline Cholesterol (at one year) & 1 & $0.96 \pm 1.10$ & 0.48 \\
\hline Diabetes (at one year) & 0.9 & $0.72 \pm 1.24$ & 0.68 \\
\hline Creatinine (at one year) & 1 & $1.00 \pm 1.00$ & 0.82 \\
\hline Hypertension (at one year) & 0.9 & $0.65 \pm 1.19$ & 0.4 \\
\hline
\end{tabular}

HLA human leucocyte antigen

after heart transplantation, are associated with a decreased long-term survival.

\section{Prevalence and predictors of CAV}

Although the donors in the Netherlands may be older than in other transplantation settings, CAV prevalence in our cohort was comparable with that presented in the most recent report of the ISHLT [1]. However, the strongest CAV risk factor in our study was older donor age. Donor age has been previously reported to be associated with an increased risk of CAV in the database of the United Network for Organ Sharing (in the United States), as well as in Spanish heart transplant registries $[15,16]$. We found that also male donor sex was associated with an increased risk of $\mathrm{CAV}$, irrespective of the gender of the recipient. Other studies have shown that gender mismatch, particularly a male recipient receiving a female donor heart, was associated with decreased survival after transplantation, but the reports are contradictory about its relationship with the risk the CAV [17]. Although we did not collect data on donor risk factors, older and male donors might reasonably have higher rates of hypertension, dyslipidaemia and/or history of smoking, conferring the higher chance of subclinical vascular atherosclerosis that might be correlated to neo-intima fibrosis in CAV. Furthermore, donor-derived immunological factors have been reported to contribute to the development of CAV in addition of recipient immunity [18].

We could not find any recipient-related independent predictors of $\mathrm{CAV}$, although in the univariate analysis the risk of CAV was significantly associated with the treatment with mycophenolate mofetil, while cytomegalovirus disease had a trend towards positive association with CAV. The role of cytomegalovirus disease in the development of CAV has 
been extensively studied, and a positive association has not always been found, but a recent Spanish report has shown that cytomegalovirus disease and even asymptomatic viraemia are associated with increased risk [3]. The possible explanations for the association between treatment with mycophenolate mofetil and CAV in the univariate analysis, but not in the multivariable analysis, could be an increased susceptibility for cytomegalovirus disease during mycophenolate mofetil treatment, or the need for increased immunosuppression due to rejection episodes. Other studies have shown that high rejection scores or repeated acute cardiac rejections are independent predictors of CAV [7, 19]. We did not find a significant association after Cox regression analysis, although the number of patients without any rejection episodes was higher in the $\mathrm{CAV}$-free cohort as compared with the CAV cohort. No other transplantation treatment was found to be associated with CAV. The use of statins has been associated with increased survival after heart transplantation, and it is recommended in the guidelines for the care of heart transplant recipients [20]. One possible explanation for the lack of a positive effect can be the inclusion of patients transplanted before the statin era in the analysis. The number of patients treated with everolimus, as maintenance therapy before coronary angiography, was too low to assess its association with CAV.

\section{Prognosis of CAV}

We present the survival curves according to the presence of $\mathrm{CAV}$ at four years after transplantation, because all patients then underwent elective coronary angiography, according to the protocol at our centre. After the fourth year, patients underwent myocardial perfusion scintigraphy yearly for evaluation of ischaemia, and coronary angiography was only performed by indication. An important finding of our study was that not only is early CAV diagnosis at four years associated with decreased long-time survival, but also that even mild coronary lesions, scored as CAV grade 1, represent a negative prognostic factor. Although the majority of studies are directed to more advanced degrees of CAV, which are also amenable to interventions, one other recent report showed that persistent mild coronary lesions have a negative prognostic impact $[2,21]$. This finding emphasises the role of preventive measures immediately after transplantation, before even mild coronary disease may develop.

\section{Limitations of the study}

This is a retrospective study, although based on a prospective cohort. The multivariate analysis of the clinical factors allows only the finding of associations with CAV, but not causal relations. Other variables potentially of interest may not have been prospectively collected or analysed. The study is limited to a single centre, and therefore other centre-related factors may prevent the generalisation of the findings.

\section{Conclusion}

Our study provides an overview of CAV prevalence and predictors in the heart transplant recipients in the Netherlands. The novel finding is that early CAV grade 1, representing mild disease, is associated with decreased longterm graft survival. We conclude that the management of CAV should start with donor selection and with preventive medical treatment according to the guidelines of transplant care, immediately after heart transplantation.

Conflict of interest G. Galli, K. Caliskan, A.H.M.M. Balk, R. van Domburg, O. Birim, J. Salerno-Uriarte, O.C. Manintveld and A.A. Constantinescu state that they have no competing interest.

Open Access This article is distributed under the terms of the Creative Commons Attribution 4.0 International License (http:// creativecommons.org/licenses/by/4.0/), which permits unrestricted use, distribution, and reproduction in any medium, provided you give appropriate credit to the original author(s) and the source, provide a link to the Creative Commons license, and indicate if changes were made.

\section{References}

1. Lund LH, Edwards LB, Kucheryavaya AY, et al. The registry of the international society for heart and lung transplantation: thirty-first official adult heart transplant report - 2014; focus theme: retransplantation. J Heart Lung Transplant. 2014;33:996-1008.

2. Agarwal S, Parashar A, Kapadia SR, et al. Long-term mortality after cardiac allograft vasculopathy: implications of percutaneous intervention. JACC Heart Fail. 2014;2:281-8.

3. Delgado JF, Reyne AG, de Dios S, et al. Influence of cytomegalovirus infection in the development of cardiac allograft vasculopathy after heart transplantation. J Heart Lung Transplant. 2015;34:1112-9.

4. Huibers MM, Vink A, Kaldeway J, et al. Distinct phenotypes of cardiac allograft vasculopathy after heart transplantation: a histopathological study. Atherosclerosis. 2014;236:353-9.

5. Huibers M, de Jonge N, van Kuik J, et al. Intimal fibrosis in human cardiac allograft vasculopathy. Transpl Immunol. 2011;25:124-32.

6. Seki A, Fishbein MC. Predicting the development of cardiac allograft vasculopathy. Cardiovasc Pathol. 2014;23:253-60.

7. Caforio AL, Tona F, Fortina $\mathrm{AB}$, et al. Immune and nonimmune predictors of cardiac allograft vasculopathy onset and severity: multivariate risk factor analysis and role of immunosuppression. Am J Transplant. 2004;4:962-70.

8. Braga JR, Santos IS, McDonald M, et al. Factors associated with the development of cardiac allograft vasculopathy - a systematic review of observational studies. Clin Transplant. 2012;26:E111-24.

9. Mehra MR, Crespo-Leiro MG, Dipchand A, et al. International Society for Heart and Lung Transplantation working formulation 
of a standardized nomenclature for cardiac allograft vasculopathy2010. J Heart Lung Transplant. 2010;29:717-27.

10. Zijlstra LE, Constantinescu AA, Manintveld O, et al. Improved long-term survival in Dutch heart transplant patients despite increasing donor age: the Rotterdam experience. Transpl Int. 2015;28:962-71.

11. Billingham ME. Diagnosis of cardiac rejection by endomyocardial biopsy. Heart Transplant. 1981;1:25-30.

12. Billingham ME, Cary NR, Hammond ME, et al. A working formulation for the standardization of nomenclature in the diagnosis of heart and lung rejection: heart rejection study group. The international society for heart transplantation. J Heart Transplant. 1990;9(6):587-93.

13. Stewart S, Winters GL, Fishbein MC, et al. Revision of the 1990 working formulation for the standardization of nomenclature in the diagnosis of heart rejection. J Heart Lung Transplant. 2005;24:1710-20.

14. Balk AH, Simoons ML, van der Linden MJ, et al. Coronary artery disease after heart transplantation: timing of coronary arteriography. J Heart Lung Transplant. 1993;12:89-99.

15. Nagji AS, Hranjec T, Swenson BR, et al. Donor age is associated with chronic allograft vasculopathy after adult heart trans- plantation: implications for donor allocation. Ann Thorac Surg. 2010;90:168-75.

16. Roig E, Almenar L, Crespo-Leiro M, et al. Heart transplantation using allografts from older donors: multicenter study results. J Heart Lung Transplant. 2015;34:790-6.

17. Khush KK, Kubo JT, Desai M. Influence of donor and recipient sex mismatch on heart transplant outcomes: analysis of the international society for heart and lung transplantation registry. J Heart Lung Transplant. 2012;31:459-66.

18. van den Hoogen P, Huibers MM, Sluijter JP, et al. Cardiac allograft vasculopathy: a donor or recipient induced pathology? J Cardiovasc Transl Res. 2015;8:106-16.

19. Guihaire J, Mercier O, Flecher E, et al. Comparison of cardiac allograft vasculopathy in heart and heart-lung transplantations: a 15year retrospective study. J Heart Lung Transplant. 2014;33:636-43.

20. Costanzo MR, Dipchand A, Starling R, et al. The international society of heart and lung transplantation guidelines for the care of heart transplant recipients. J Heart Lung Transplant. 2010;29:914-56.

21. Zakliczynski M, Babinska A, Flak B, et al. Persistent mild lesions in coronary angiography predict poor long-term survival of heart transplant recipients. J Heart Lung Transplant. 2014;33:618-23. 